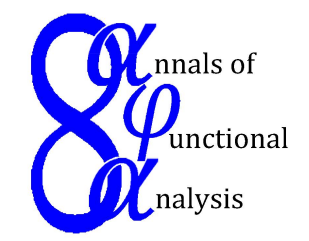

Ann. Funct. Anal. 6 (2015), no. 1, 1-11

http://doi.org/10.15352/afa/06-1-1

ISSN: 2008-8752 (electronic)

http://projecteuclid.org/afa

\title{
A BURENKOV'S TYPE RESULT FOR FUNCTIONS OF BOUNDED $\kappa$-VARIATION
}

\author{
JOSÉ GIMÉNEZ ${ }^{*}$, LORENA LÓPEZ ${ }^{1}$, NELSON MERENTES² AND J. L. SÁNCHEZ ${ }^{2}$ \\ Communicated by V. Valov
}

\begin{abstract}
In this paper, we give a sufficient condition for a linear composition operator to map the space of functions of bounded Koremblum variation, $\kappa B V[a, b]$, into itself. We present several results concerning quasi monotonic properties of the functionals of $\kappa$-variation and prove a Burenkov's type result for functions belonging to $\kappa B V[a, b]$.
\end{abstract}

\section{INTRODUCTION AND PRELIMINARIES}

The class of all real valued functions (defined on an closed real interval) of bounded variation was introduced by C. Jordan in 1881 ([9]), who established the relation between these and the class of all monotone functions; namely,

A function $f$ is of bounded variation if and only if it is the difference of two monotone functions.

This fact has many important implications For instance, every function of bounded variation, may only have jump discontinuities; on the other hand, a function of bounded variation is differentiable almost everywhere (a celebrated Theorem of H. Lebesgue).

Historically one of the most important implications of Jordan's characterization is that it permits to extend Dirichlet's criterium (for the convergence of the Fourier series of piecewise monotone functions) to the class of functions of bounded variation.

In order to recall the basic definitions, we first introduce some notations.

Date: Received: Jan. 5, 2013; Accepted: Mar. 9, 2013.

* Corresponding author.

2010 Mathematics Subject Classification. Primary 26A45; Secondary 47H30.

Key words and phrases. Composition operator, distortion function, function of bounded $\kappa$ variation. 
Given two sets $A$ and $B$, the notation $A^{B}$ stands for the set of all functions from $B$ to $A$. Given a closed interval $[a, b] \subset \mathbb{R}$ and a function $f \in \mathbb{R}^{[a, b]}$, if $I=[c, d] \subset[a, b]$, we will use the following notations:

$$
\begin{gathered}
|I|:=d-c \\
f[I]:=f(d)-f(c) . \\
|f[I]|:=|f(d)-f(c)| .
\end{gathered}
$$

The notation $\pi[a, b]$ will be used for the set of all partitions $\xi=\left\{t_{i}\right\}_{i=0}^{n}$ of $[a, b]$ : i.e., $n$ is some positive integer and $a=t_{0}<t_{1}<\cdots<t_{n}=b$. When it is clear from the context that we refer to a given partition $\xi$ we will also write $\xi=\left\{I_{j}\right\}_{1}^{n}$, where $I_{j}=I_{j}(\xi):=\left[t_{j-1}, t_{j}\right] \quad(j=1,2, \ldots, n)$.

A function $f:[a, b] \rightarrow \mathbb{R}$ is said to be of bounded variation, on $[a, b]$, if there is a constant $M>0$ such that

$$
\sum_{j=1}^{n}\left|f\left[I_{j}\right]\right| \leq M
$$

for all $\xi=\left\{I_{j}\right\}_{1}^{n} \in \pi[a, b]$.

The total variation of $f$ on $[a, b]$, is denoted as $V(f ;[a, b])$, or simply by $V(f)$, and it is the supremum of the sums (1.1) over $\pi[a, b]$.

We will call a sum such as (1.1) a Jordan's sum and denote it as $\Sigma(f ; \xi)$.

The unvarying interest generated by the classical notion of function of bounded variation has lead to some generalizations of the concept, mainly, intended to the search of bigger classes of functions whose elements have pointwise convergent Fourier series, see e.g., [5, 14, 15, 16, 17, 18]. As in the classical case, these generalizations have also found many applications in the study of certain (partial) differential and integral equations (see e.g., [3]) and also in the theory of linear and nonlinear composition operators; namely, to find necessary and sufficient conditions guaranteeing that such an operator maps a given space of functions of generalized bounded variation (or a subset of it) into itself. Other conditions, called acting conditions involve: boundedness, continuity, compactness (in the linear case), to satisfy a local or global Lipschitz condition, etc., see e.g., [1, 6, 8, $10]$.

In 1975, B. Korenblum [12], introduced the notion of $\kappa$-variation of a function, while studying the problem of representation of harmonic functions defined on the unit disk of the complex plane by means of generalized Poisson integrals involving, so-called, premeasures defined on sub-intervals of $[0,2 \pi]$. This notion differs from the classical notion, and other known variations, in that Korenblum's concept maximizes ratios between Jordan's sums and the so-called $\kappa$-entropies generated by a distortion function ${ }^{1} \kappa$, which measures lengths in the domain of the functions. A weak point of this notion, as we will show later, is that the associated $\kappa$-variation functionals need not be monotone with respect to enlargements of partitions or additive on union of intervals. An advantage, on the other hand, is that a function of bounded $\kappa$-variation can be decomposed into the difference of two, so called, $\kappa$-decreasing functions (see [7]).

\footnotetext{
${ }^{1}$ Actually the letter $\kappa$ stands for Carleson, according to Korenblum, [12].
} 
Definition 1.1. A function $\kappa:[0,1] \longrightarrow[0,1]$, is called a distortion function if it is continuous, nondecreasing, concave on $[0,1]$ and such that $\kappa(0)=0, \kappa(1)=1$, and

$$
\lim _{x \rightarrow 0^{+}} \frac{\kappa(x)}{x}=+\infty
$$

That is, $\kappa$ have infinite slope at the origin.

Note that any distortion function $\kappa$ satisfies

$$
\frac{\kappa(x+y)-\kappa(y)}{(x+y)-y} \leq \frac{\kappa(x)-\kappa(0)}{x-0} \quad(x, y, x+y \in[0,1])
$$

and therefore it is subadditive; that is, if $x, y \in[0,1]$ are such that $x+y \in[0,1]$ then

$$
\kappa(x+y) \leq \kappa(x)+\kappa(y)
$$

in particular, if $x \in[0,1]$ and $n, m \in \mathbb{N}, \mu \in[0,+\infty)$ are such that $n x \in$ $[0,1], \mu x \in[0,1]$ then

$$
\frac{1}{m} \kappa(x) \leq \kappa\left(\frac{x}{m}\right), \quad \kappa(n x) \leq n \kappa(x) \quad \text { and } \quad \kappa(\mu x) \leq[[\mu+1]] \kappa(x),
$$

where $[[a]]$ denotes the integer part of a positive real number $a$; that is,

$$
[[a]]:=\max \{n \in \mathbb{N}: n \leq a\} .
$$

The set of all distortion functions will be denoted as $\mathcal{K}$.

Throughout the paper, unless explicitly stated otherwise, we will assume that $\kappa$ is a distortion function and $[a, b] \subset \mathbb{R}$ is a closed interval.

Definition 1.2. ([13]) Let $\kappa \in \mathcal{K}$ and let $\xi=\left\{I_{j}\right\}_{1}^{n} \in \pi[a, b]$. The quantity

$$
\kappa(\xi):=\kappa(\xi ;[a, b])=\sum_{j=1}^{n} \kappa\left(\frac{\left|I_{j}\right|}{b-a}\right)
$$

is called the $\kappa$-entropy of $\xi$, relative to $[a, b]$.

Examples ([13]):

- $\kappa(s):=s(1-\log s)$. The corresponding entropy is called the Shannon entropy.

- $\kappa(s):=s^{\alpha} \quad(0<\alpha<1)$. The corresponding entropy is called the Lipschitz entropy.

- $\kappa(s):=\left(1-\frac{1}{2} \log s\right)^{-1}$. The corresponding entropy is called the Dini entropy.

Notice that for any $\xi=\left\{t_{i}\right\}_{i=0}^{n} \in \pi[a, b]$ we have

$$
1=\kappa(1)=\kappa\left(\sum_{i=1}^{n} \frac{t_{i}-t_{i-1}}{b-a}\right) \leq \kappa(\xi) .
$$

Let $\kappa \in \mathcal{K}$. A function $f \in \mathbb{R}^{[a, b]}$ is said to be of bounded $\kappa$-variation if there is a positive constant $\mathrm{C}$ such that, for every partition $\xi$ of $[a, b]$,

$$
\Sigma(f ; \xi) \leq C \kappa(\xi ;[a, b]) .
$$


The total $\kappa$-variation of $f$ in $[a, b]$ is defined as

$$
\kappa V(f)=\kappa V(f,[a, b]):=\inf \{C: \Sigma(f ; \xi) \mid \leq C \kappa(\xi ;[a, b]): \xi \in \pi[a, b]\} .
$$

The set of all functions of bounded $\kappa$-variation on $[a, b]$ will be denoted by $\kappa B V[a, b]$. It is readily seen that this is a linear space. Equipped with the norm

$$
\|f\|_{\kappa B V[a, b]}:=|f(a)|+\kappa V(f ;[a, b])
$$

the space $\kappa B V[a, b]$ is a Banach space (see $[2,11]$ ); in fact, it readily follows from the definitions that if $f, g \in \kappa B V[a, b]$ then

$$
\|f g\|_{\kappa B V[a, b]} \leq\|f\|_{\infty}\|g\|_{\kappa B V[a, b]}+\|g\|_{\infty}\|f\|_{\kappa B V[a, b]} .
$$

Considering the partition $\xi:=\{a, s, b\}$, for fixed $s$, it is readily seen that every $f \in \kappa B V[a, b]$ is bounded with

$$
\|f\|_{\infty} \leq 2\|f\|_{\kappa B V}
$$

On the other hand, from (1.3) it is quite easy to show that every function of bounded (Jordan) variation on $[a, b]$ is in $\kappa B V[a, b]$, and

$$
\kappa V(f) \leq V(f)
$$

Also, from (1.3) and the fact that the trivial partition $\{a, b\}$ is optimum for (1.4), it follows that if $f$ is a monotone function on $[a, b]$ then

$$
\kappa V(f)=V(f)=|f(b)-f(a)| .
$$

In his seminal 1975 paper, [12, Theorem 4.2], B. Korenblum proves a canonical decomposition theorem for the functions in the space $\kappa B V[a, b]$, when $\kappa$ is the Shannon distortion. Later, Cyphert and Kelingos ([7]) generalized that result by showing, for any $\kappa \in \mathcal{K}$, that every function in $\kappa B V[a, b]$ can be expressed as the difference of two $\kappa$-decreasing functions.

A function $f \in \mathbb{R}^{[a, b]}$ is said to be $\kappa$-decreasing if there is a constant $A \geq 0$ such that for every interval $I=[x, y] \subset[a, b]$,

$$
f(y)-f(x) \leq A k\left(\frac{y-x}{b-a}\right)
$$

From this decomposition theorem it follows that every function $f \in \kappa B V[a, b]$ is regulated; that is, it has one sided limits $f(t+)$ y $f(t-)$ at every point $t \in(a, b)$ and the limits $f(a+)$ and $f(b-)$ exist. Thus, we have the following chain of inclusions

$$
B V[a, b] \subseteq \kappa B V[a, b] \subseteq \mathfrak{R}[a, b]
$$

where $\mathfrak{R}[a, b]$ denote the set of all regulated functions on $[a, b]$.

That the inclusions in (1.9) are strict can be seen, for instance, in [1, 7]. 


\section{QuASI-MONotonic PROPERTIES OF The FUnCtionals $\kappa V(f ; \cdot)$}

As mentioned above, a weak point of the notion of (bounded) $\kappa$-variation, is that the $\kappa$-variation functional associated to it, need not be monotone with respect to enlargements of partitions or additive on union of intervals.

In [7], the following example was given to show that the functional $\kappa V(f ; x):=$ $\kappa V(f ;[0, x])$,

$x \in(0,1]$, need not be increasing with $\mathrm{x}$.

For the sake of notational simplicity, we will also denote by $\kappa V(f ; \cdot)$, the partitions functional

$$
\kappa V(f ; \xi)=\kappa V(f ; \xi,[a, b]):=\frac{\Sigma(f ; \xi)}{\kappa(\xi ;[a, b])},
$$

where $f \in \mathbb{R}^{[a, b]}$ and $\xi=\left\{I_{j}\right\}_{1}^{n} \in \pi[a, b]$.

Fix $0<a<1$ and $0 \leq b \leq 1$. Let $f$ be the continuous function which is linear on the intervals $[0, a]$ and $[a, 1]$ and such that $f(0)=0, f(a)=1$ and $f(1)=b$. Then, from the piecewise linearity of $f$ and the subadditivity of $\kappa$ it follows that

$$
\kappa V(f)=\max \left\{b, \frac{2-b}{\kappa(a)+\kappa(1-a)}\right\} .
$$

Choose $\frac{2}{\kappa(a)+\kappa(1-a)}<b<1$. Let $\xi:=\{0,1\}$ and let $\eta:=\{0, a, 1\}$. Then

$$
\kappa V(f ; \xi)=b>\frac{2-b}{\kappa(a)+\kappa(1-a)}=\kappa V(f ; \eta)
$$

therefore, $\kappa V(f ; \cdot)$ need not be increasing with respect to partition enlargements.

On the other hand, from (1.7) it follows that $\kappa V(f ;[0, a])=1$ and $V(f ;[a, 1])=$ $1-b$. Hence

$$
\kappa V(f ;[0, a])+V(f ;[a, 1])=2-b>b=V(f ;[0,1]) .
$$

This shows (for $b$ as above) that $\kappa V(f ;)$ need not be additive on union of intervals.

In the rest of this section we examine what we may call quasi-monotonic properties of the functionals $\kappa V(f ; \cdot)$. The designation "quasi-monotonic" refers to the fact that given two real valued functionals $A, B$ defined on a subspace $X \subset \mathbb{R}^{[a, b]}$, there is a positive number $M$ such that $A(f) \leq M B(f)$, for all $f \in X$.

Theorem 2.1. Suppose $f \in \kappa B V[0,1]$. Then, for all $[a, b] \subseteq[0,1],\left.\quad f\right|_{[a, b]} \in$ $\kappa B V([a, b]$ and

$$
\kappa V\left(\left.f\right|_{[a, b]} ;[a, b]\right) \leq\left(\frac{\kappa(a)+\kappa(1-b)}{\kappa(b-a)}+1\right) \kappa V(f ;[0,1]) .
$$

Proof. Let $\xi=\left\{t_{i}\right\}_{0}^{n}=\left\{I_{i}\right\}_{1}^{n} \in \pi[a, b]$ and set $\xi^{\prime}=\xi \cup\{0\} \cup\{1\}$.

First of all, notice that $\kappa\left(\frac{x-y}{b-a}\right) \geq \kappa(x-y)$ (since $b-a<1$, for all $x, y \in[a, b]$ with $x>y$ ). Thus

$$
\kappa V\left(\left.f\right|_{[a, b]} ; \xi\right)=\frac{\Sigma(f ; \xi)}{\kappa(\xi)} \leq \frac{\Sigma(f ; \xi)}{\sum_{i=1}^{n} \kappa\left(\left|I_{i}\right|\right)} .
$$


Notice also that $\sum_{i=1}^{n} \kappa\left(\left|I_{i}\right|\right) \geq \kappa\left(\sum_{i=1}^{n}\left|I_{i}\right|\right)=\kappa(b-a)$. Let $\lambda:=$ $\frac{\kappa(a)+\kappa(1-b)}{\kappa(b-a)}$. Then (2.1) implies that

$$
\begin{aligned}
& \kappa V\left(\left.f\right|_{[a, b]} ; \xi\right) \leq \frac{(\lambda+1)}{(\lambda+1)} \frac{\sum(f ; \xi)}{\sum_{i=1}^{n} \kappa\left(\left|I_{i}\right|\right)} \\
= & \frac{(\lambda+1) \Sigma(f ; \xi)}{\sum_{i=1}^{n} \kappa\left(\left|I_{i}\right|\right)+\lambda \sum_{i=1}^{n} \kappa\left(\left|I_{i}\right|\right)} \\
\leq & \frac{(\lambda+1) \Sigma(f ; \xi)}{\sum_{i=1}^{n} \kappa\left(\left|I_{i}\right|\right)+\kappa(a)+\kappa(1-b)} \\
\leq & \frac{(\lambda+1)\left(\sum(f ; \xi)+|f(a)-f(0)|+|f(1)-f(b)|\right)}{\sum_{i=1}^{n} \kappa\left(\left|I_{i}\right|\right)+\kappa(a)+\kappa(1-b)} \\
= & (\lambda+1) \kappa V\left(f ; \xi^{\prime}\right) .
\end{aligned}
$$

Therefore

$$
\kappa V\left(\left.f\right|_{[a, b]} ;[a, b]\right) \leq\left(\frac{\kappa(a)+\kappa(1-b)}{\kappa(b-a)}+1\right) \kappa V(f,[0,1]) .
$$

Corollary 2.2. Let $f \in \kappa B V[0,1]$ and let $\xi=\left\{x_{i}\right\}_{0}^{n} \in \pi[a, b]$, where $[a, b] \subset[0,1]$. For all $i=1,2, \ldots, n$, put $L_{i}:=\left[x_{i-1}, x_{i}\right]$. Then

$$
\sum_{i=1}^{n} \kappa V\left(\left.f\right|_{L_{i}}, L_{i}\right) \leq M_{\kappa}(\xi) \kappa V(f,[0,1])
$$

where $M_{\kappa}(\xi)=n+\sum_{i=1}^{n}\left(\frac{\kappa\left(x_{i-1}\right)+\kappa\left(1-x_{i}\right)}{\kappa\left(\left|L_{i}\right|\right)}\right)$.

Theorem 2.3. Let $f \in \kappa B V[0,1]$ and let $\xi=\left\{x_{i}\right\}_{0}^{n} \in \pi[0,1]$. For all $i=$ $1,2, \ldots, n$, put $L_{i}:=\left[x_{i-1}, x_{i}\right]$. Then

$$
\kappa V(f,[0,1]) \leq N_{\kappa}(\xi) \sum_{i=1}^{n} \kappa V\left(\left.f\right|_{L_{i}} ; L_{i}\right),
$$

where $N_{\kappa}(\xi)=3 \max _{i}\left\{\left[\left[\left(\left(x_{i}-x_{i-1}\right)^{-1}+1\right)\right]\right]\right\}$.

Proof. Let $\eta=\left\{y_{j}\right\}_{0}^{m} \in \pi[0,1]$.

For each $i=1,2, \ldots, n$, put $\eta_{i}:=\eta \bigcap L_{i}=\left\{y_{j}^{i}\right\}_{0}^{n_{i}}$.

It is clear that $\eta_{i}$ need not be a partition of $L_{i}$, since, except 0 and 1 , some of the points $x_{1}, x_{2}, \ldots, x_{n-1}$ might not belong to $\eta$. To get partitions in each $L_{i}$, set

$$
\eta_{i}^{\prime}:=\eta_{i} \bigcup\left\{x_{i-1}, x_{i}\right\}=\left\{z_{j}^{i}\right\}_{0}^{n_{i}^{\prime}}, \quad i=1,2, \ldots, n .
$$

Notice that if $\eta_{i}=\emptyset$, then

$$
\kappa V\left(f ; \eta_{i}^{\prime}, L_{i}\right):=\left|f\left(x_{i}\right)-f\left(x_{i-1}\right)\right| .
$$


Clearly $\eta^{\prime}:=\bigcup_{i=1}^{n} \eta_{i}^{\prime} \in \pi[a, b]$, and (since Jordan's sums are increasing with respect to refinements of partitions)

$$
\kappa V(f ; \eta,[0,1])=\frac{\Sigma(f ; \eta)}{\kappa(\eta)} \leq \frac{\Sigma\left(f ; \eta^{\prime}\right)}{\kappa(\eta)}=\sum_{i=1}^{n} \frac{\Sigma\left(\left.f\right|_{L_{i}} ; \eta_{i}^{\prime}\right)}{\kappa(\eta)}
$$

Now suppose that for some $i \in\{1,2, \ldots, n\} \eta_{i} \neq \emptyset$ and that, in the worst case scenario, neither $x_{i-1}$ nor $x_{i}$ are in $\eta_{i}$. Then, if $\lambda_{i}:=\kappa\left(x_{i}-y_{n_{i}}^{i}\right)+\kappa\left(y_{0}^{i}-x_{i-1}\right)$, the fact that $\kappa(\eta)$ satisfies

$$
\kappa(\eta) \geq 1 \quad \text { and } \quad \kappa(\eta) \geq \sum_{j=1}^{n_{i}} \kappa\left(y_{j}^{i}-y_{j-1}^{i}\right)
$$

implies

$$
3 \kappa(\eta) \geq\left(\lambda_{i}+1\right) \kappa(\eta)=\lambda_{i} \kappa(\eta)+\kappa(\eta) \geq \lambda_{i}+\kappa(\eta) \geq \sum_{j=1}^{n_{i}^{\prime}} \kappa\left(z_{j}^{i}-z_{j-1}^{i}\right) .
$$

And, from (1.2), we obtain

$$
\sum_{j=1}^{n_{i}^{\prime}} \kappa\left(z_{j}^{i}-z_{j-1}^{i}\right) \geq \sum_{j=1}^{n_{i}^{\prime}}\left[\left[\left(\left(x_{i}-x_{i-1}\right)^{-1}+1\right)\right]\right]^{-1} \kappa\left(\frac{z_{j}^{i}-z_{j-1}^{i}}{x_{i}-x_{i-1}}\right) \geq m \kappa\left(\eta_{i}^{\prime}, L_{i}\right)
$$

where $m:=\min \left\{\left[\left[\left(\left(x_{i}-x_{i-1}\right)^{-1}+1\right)\right]\right]^{-1}: i=1,2, \ldots, n\right\}$.

Taking into account the fact that that $\frac{3}{m} \geq 1,(2.2),(2.3),(2.4)$ and $(2.5)$, we get

$$
\kappa V(f ; \eta,[0,1]) \leq \sum_{i=1}^{n} \frac{3}{m} \frac{\Sigma\left(\left.f\right|_{L_{i}} ; \eta_{i}^{\prime}\right)}{\kappa\left(\eta_{i}^{\prime}, L_{i}\right)} \leq \frac{3}{m} \sum_{i=1}^{n} \kappa V\left(\left.f\right|_{L_{i}} ; L_{i}\right)
$$

which completes the proof.

The same proof of Theorem 2.3 may be used to prove the following result.

Corollary 2.4. Let $f \in \mathbb{R}^{[0,1]}$ and suppose that $f$ is of bounded $\kappa$-variation on every (closed) interval determined by a fixed partition $\xi=\left\{L_{i}\right\}$. Then $f \in$ $\kappa V B[0,1]$ and $\kappa V(f,[0,1]) \leq N \sum_{i=1}^{n} \kappa V\left(\left.f\right|_{L_{i}} ; L_{i}\right)$, where $N$ is a constant depending only on $\xi$.

Proof. Indeed, in this case the result follows from (2.6) and the facts that the partition $\eta$ is arbitrary and $f$ is of bounded $\kappa$-variation on each $L_{i}$.

Clearly, we can easily adapt the proofs of Theorems 2.1 and 2.3 to obtain versions of them for arbitrary closed intervals.

Corollary 2.5. Let $f \in \mathbb{R}^{[a, b]}$ and suppose that $[a, b]$ can be decomposed into finitely many closed intervals on which $f$ is monotone. Then $f \in \kappa V B[a, b]$.

Proof. It is immediate from Corollary 2.4 and (1.9). 


\section{Composing Functions of Bounded $\kappa$-VARiation}

Let $\mathbb{X}$ be a subspace of $\mathbb{R}^{[a, b]}$. Given a function $g: \mathbb{R} \rightarrow \mathbb{R}$, the autonomous (non-linear) superposition operator $S_{g}: \mathbb{X} \rightarrow \mathbb{R}^{[a, b]}$, generated by $g$, is defined as

$$
S_{g}(f)(t):=(g \circ f)(t)=g(f(t))(t \in[a, b]) .
$$

In [1] J. Appel and N. Merentes, show that, in general, the composition of two functions of bounded $\kappa$-variation need not be of bounded $\kappa$-variation. Moreover, they show that a superposition operator, $S_{h}$, maps the space $\kappa V B[a, b]$ into itself if and only if the function $h$ satisfies a local Lipschitz condition.

A linear composition operator, on the other hand, is defined as follows:

Suppose that $D$ and $E$ are given sets, $X$ is a linear subspace of $\mathbb{R}^{E}$ and $f$ is a map from $D$ to $E$, the composition operator $C_{f}: X \rightarrow \mathbb{R}^{D}$ is defined by

$$
C_{f}(g):=g \circ f .
$$

Despite the obvious differences in acting conditions of these operators, they share some attributes and it often happens (see e.g, [4, 8]) that certain properties of one them can be deduced from the properties of the other.

In our next result we give a sufficient condition for an operator $C_{f}$ to map $\kappa B V[a, b]$ into $\kappa B V[c, d]$. Recall that a function $f:[c, d] \rightarrow \mathbb{R}$ is said to be Lipschitz continuous iff

$$
L(f):=\sup \left\{\frac{f(x)-f(y)}{x-y}: x, y \in[c, d], x \neq y\right\}<\infty .
$$

Theorem 3.1. Suppose that $f:[c, d] \rightarrow[a, b]$ is an injective Lipschitz continuous function. Then $C_{f}$ maps $\kappa B V[a, b]$ into $\kappa B V[c, d]$ and is bounded (continuous).

Proof. It follows from the hypothesis that $f$ is continuous and injective, hence it is strictly monotonic on $[c, d]$. Assume, that $f$ is increasing and that $g \in \kappa B V[a, b]$.

Let $\xi=\left\{t_{i}\right\}_{0}^{n}=\left\{I_{i}\right\}_{1}^{n} \in \pi[c, d]$. Since $f$ is Lipschitz, there is a real number $L \geq 0$ such that $f(y)-f(x) \leq L(y-x)$ for all $x, y \in[c, d]$, such that $x<y$. Hence, for each $i=1,2, \ldots, n$

$$
\begin{aligned}
\frac{\left|f\left[I_{i}\right]\right|}{b-a} & \leq \frac{L(d-c)}{(b-a)} \frac{\left|I_{i}\right|}{(d-c)} \\
& \leq\left(\left[\left[L(d-c)(b-a)^{-1}\right]\right]+1\right) \frac{\left|I_{i}\right|}{(d-c)} \\
& =N\left(\frac{\left|I_{i}\right|}{d-c}\right)
\end{aligned}
$$

where $N:=\left[\left[L(d-c)(b-a)^{-1}\right]\right]+1$.

It follows from the monotonicity and subadditivity of $\kappa$ that

$$
\begin{aligned}
\kappa\left(\frac{\left|I_{i}\right|}{(d-c)}\right) & \geq \kappa\left(\frac{1}{N} \frac{f\left[I_{i}\right]}{b-a}\right) \\
& \geq \frac{1}{N} \kappa\left(\frac{f\left[I_{i}\right]}{b-a}\right)
\end{aligned}
$$


Hence

$$
\kappa V(g \circ f, \xi) \leq N \frac{\sum_{i=1}^{n} \mid\left(g\left(f\left(t_{i}\right)\right)-g\left(f\left(t_{i-1}\right)\right) \mid\right.}{\sum_{i=1}^{n} \kappa\left(\frac{f\left(t_{i}\right)-f\left(t_{i-1}\right)}{b-a}\right)} .
$$

By using a " $\lambda+1$-argument" as in the proofs of Theorems 2.1 and 2.3, with

$$
\lambda:=\kappa\left(\frac{f(c)-a}{b-a}\right)+\kappa\left(\frac{b-f(d)}{b-a}\right),
$$

we obtain

$$
\kappa V(g \circ f, \xi) \leq(\lambda+1) N \kappa V(g, \eta) \leq(\lambda+1) N \kappa V(g ;[a, b])
$$

where $\eta=\left\{f\left(t_{i}\right)\right\}_{i=0}^{n} \cup\{a, b\}$.

Therefore,

$$
\kappa V(g \circ f ;[c, d]) \leq(\lambda+1) N \kappa V(g ;[a, b]) .
$$

From (3.1) it follows that $C_{f}$ maps $\kappa B V[a, b]$ into $\kappa B V[c, d]$ and that there is a real number $M \geq 0$ such that

$$
\left\|C_{f}(g)\right\| \leq M\|g\|
$$

for all $g \in \kappa B V[a, b]$; that is, $C_{f}$ is bounded.

The case in which $f$ decreasing is treated similarly.

Notice that if $f$ is as in the statement of Theorem 3.1 then it is a $\kappa$-decreasing function (cf. (1.8)).

As mentioned earlier, the property of functions in $\mathbb{R}^{[a, b]}$ of being of bounded $\kappa$-variation is not closed under compositions. However, the fact that $\kappa V B[a, b]$ is an algebra, suggest us to look for variations of the following procedure:

Suppose that $D$ and $E$ are given sets, $X$ is a linear subspace of $\mathbb{R}^{E}$ and $f$ is a map from $D$ to $E$. By enhancing the intrinsic properties (smoothness) of the inner function $f$, we expect that the acting properties of the linear composition operator $C_{f}$ will actually improve, and this improvement should be transferred to a product of the form $C_{f}(g) \cdot h$, where $g, h \in X$. A common instance, when the spaces under consideration are algebras, is to ask that the function $f$ has a derivative, $f^{(k)}, k \in \mathbb{N}$, which is also in the algebra, and consider the product $(g \circ f) f^{(k)}$.

The following result, due to V. I. Burenkov for the case of the algebra $B V[a, b]$, illustrated this fact. For the proof the reader is referred to [4, Theorem 5] (see also [8]).

Theorem 3.2 (Burenkov). Suppose that $f$ has a derivative $f^{(k)}$ of order $k$ everywhere on $[a, b]$. If $f^{(k)} \in B V[a, b]$ and if $g \in B V[c, d], c:=\min _{[a, b]} f, d:=$ $\max _{[a, b]} f$, then the function $(g \circ f) f^{(k)}$ is also of bounded variation on $[a, b]$; moreover, there is number $M=M(k) \geq 0$ such that

$$
\left\|(g \circ f) f^{(k)}\right\|_{B V[a, b]} \leq M\|g\|_{B V[c, d]}\left\|f^{(k)}\right\|_{B V[a, b]} .
$$


In order to get a version of Burenkov's result for functions of $\kappa$-bounded variation, we will need to impose additional conditions to the inner function of a composition $g \circ f$.

Recall that the support of a real valued function is the set of points where the function is not zero. We will say that a function $f \in \mathbb{R}^{[a, b]}$ has cofinite support if the set $\{x \in[a, b]: f(x)=0\}$ is finite.

By $\kappa B V_{C S}[a, b]$ we will designate the set of all functions in $\kappa B V[a, b]$ with cofinite support. Notice that this is a large subset (actually a cone) of $\kappa B V[a, b]$ since it contains all polynomials.

Theorem 3.3. Suppose that $f$ has a derivative $f^{(k)}$ of order $k$ everywhere on $[a, b]$. If $f^{(k)} \in \kappa B V_{C S}[a, b]$ and if $g \in \kappa B V[c, d], c:=\min _{[a, b]} f, d:=\max _{[a, b]} f$, then the function $(g \circ f) f^{(k)}$ is of bounded $\kappa$-variation on $[a, b]$; moreover, there is a number $M=M(k) \geq 0$ such that

$$
\left\|(g \circ f) f^{(k)}\right\|_{\kappa B V[a, b]} \leq M\|g\|_{\kappa B V[c, d]}\left\|f^{(k)}\right\|_{\kappa B V[a, b]} .
$$

Proof. The hypothesis on $f^{(k)}$ imply that we can express the set $S=\operatorname{supp}\left(f^{(k)}\right)$ as a finite union of intervals, say $S=\cup_{i=1}^{n}\left(a_{i}, b_{i}\right)$, where $a_{i}<b_{i}$ and $n \in \mathbb{N}$. Now, since $f^{(k)} \neq 0$ on $S$, given $i \in\{1, \ldots, n\}$ there is a nonnegative integer $m_{i} \leq k$ such that $\left[a_{i}, b_{i}\right]$ can be decomposed into $m_{i}$ intervals, $\left[a_{i}^{1}, b_{i}^{1}\right],\left[a_{i}^{2}, b_{i}^{2}\right], \ldots,\left[a_{i}^{m_{i}}, b_{i}^{m_{i}}\right]$, on which $f$ is strictly monotone (this follows from finitely many successive applications of Rolle's Theorem). Since $f^{\prime}$ is bounded, it is monotone and Lipschitz continuous on each $\left[a_{i}^{j}, b_{i}^{j}\right], j=1,2, \ldots, m_{i}$. It follows from Theorem 3.1 that for each $i=1,2, \ldots, n, \quad j=1,2, \ldots, m_{i}$

$$
\left.g \circ f\right|_{\left[a_{i}^{j}, b_{i}^{j}\right]} \in \kappa B V[a, b]
$$

and that there are constants $N_{i}$ such that

$$
\|g \circ f\|_{\kappa B V\left(\left[a_{i}^{j}, b_{i}^{j}\right]\right)} \leq N_{i}\|g\|_{\kappa B V\left(f\left(\left[a_{i}^{j}, b_{i}^{j}\right]\right)\right)} .
$$

From this fact, we conclude, after successive applications of Corollary 2.4 and Corollary 2.2 that $g \circ f \in \kappa B V[a, b]$ and

$$
\|g \circ f\|_{\kappa B V([a, b])} \leq M\|g\|_{\kappa B V[c, d]}
$$

for some constant $M$.

The estimate (3.2) then follows from (1.6) and (1.5).

By using an argument similar to the one given in the proof of Theorem 3.3 we obtain the following result. We omit the details of the proof.

Theorem 3.4. Suppose that $P:[c, d] \rightarrow[a, b]$ is a polynomial. Then $C_{p}$ maps $\kappa B V[a, b]$ into $\kappa B V[c, d]$ and is bounded.

Acknowledgement. This research has been partly supported by the Central Bank of Venezuela. We want to give thanks to the library staff of B.C.V. for compiling the references.

The authors would like to express their gratitude to the referee of the first version of this paper who made many valuable suggestions that help to improve the presentation of the paper. 


\section{REFERENCES}

1. J. Appell and N. Merentes, Composing Functions of Bounded Korenblum Variation, Dynam. Systems Appl. 22 (2013), no. 2-3, 197-206.

2. W. Aziz, J. A. Guerrero, J.L. Sanchez and M. Sanoja, Lipschitzian Composition Operator in the Space $\kappa B V[a, b]$, J. Math. Control Sci Appl. 4 (2011), no. 1, 67-73.

3. D. Bugajewska, D. Bugajewski and G. Lewicki, On nonlinear integral equations in the space of functions of bounded generalized $\varphi$-variation, J. Integral Equations Appl. 21 (2009), no. $1,1-20$.

4. V.I. Burenkov, On integration by parts and a problem on composition of absolutely continuous functions which arises in this connection, Theory of functions and its applications, Trudy Mat. Inst. Steklov. 134 (1975), 38-46.

5. V.V. Chistyakov, On mappings of bounded variation, J. Dynam. Control Systems 3 (1997), no. 2, 261-289.

6. V.V. Chistyakov, Mappings of generalized variation and composition operators, J. Math. Sci. 110 (2002), 2455-2466.

7. D.S. Cypher and J.A. Kelingos, The decomposition of functions of bounded k-variation into diffences of k-decresaing functions, Studia Math. 81 (1985), 185-195.

8. J. Giménez, L. López and N. Merentes, The Nemitskij operator on Lip ${ }^{k}$-type and AC ${ }^{k}$-type spaces, Demonstratio Math. 46 (2013), no. 3, 543-558.

9. C. Jordan, Sur la Série de Fourier, C. R. Acad. Sci. Paris 2 (1881), 228-230.

10. M. Josephy, Composing functions of bounded variation, Proc. Amer. Math. Soc. 83 (1981), no. $2,354-356$.

11. S.K. Kim and J. Yoon, Riemman-Stieltjes Integral of Functions of $\kappa$-bounded Variation, Comm. Korean Math. Soc. 5 (1990), no. 2, 65-73.

12. B. Korenblum, An extension of the Nevalinna theory, Acta Math. 135 (1975), 187-219.

13. B. Korenblum, A generalization of two classical convergence tests for Fourier series and some new Banach spaces of functions, Bull. Amer. Math. Soc. 9, no 2, 15-18.

14. F. Riesz, Sur certains systems singuliers d'equations integrates, Annales de L'Ecole Norm. Sup., Paris 3 (1911), no. 28, 33-68.

15. M. Schramm, Functions of $\Phi$-bounded variation and Riemann-Stieltjes Integration, Trans. Amer. Math. Soc. 287 (1985), 49-63.

16. D. Waterman, On convergence of Fourier series of functions of generalized bounded variation, Studia Math. 44 (1972), 107-117.

17. N. Wiener, Sur une generalisation de la notion de variation de pussance p-iéme bornée au sens de M. Wiener, et sur la convergence des series de Fourier, C. R. Acad. Sci. Paris 204 (1937), Ser A-B, 470-472.

18. L.C. Young, The quadratic variation of a function and its Fourier coefficients, J. Mass. Inst. Technology 3 (1924), 73-94.

1 Universidad de Los Andes, Facultad de Ciencias, Dpto. de Matemáticas, MÉRIDA-VENEZUELA.

E-mail address: jgimenez@ula.ve; lomalopez@ula.ve

${ }^{2}$ Universidad Central de Venezuela, Escuela de Matemáticas, Caracas-Venezuela

E-mail address: nmerucv@gmail.com; jose.sanchez@ciens.ucv.ve 